\title{
A MEASUREMENT OF THE CMB 〈EE〉 SPECTRUM FROM THE 2003 FLIGHT OF BOOMERANG
}

T. E. Montroy, ${ }^{1}$ P. A. R. Ade, ${ }^{2}$ J. J. Bock,${ }^{3,4}$ J. R. Bond,${ }^{5}$ J. Borrill, ${ }^{6,7}$ A. Boscaleri, ${ }^{8}$ P. Cabella, ${ }^{9}$ C. R. Contaldi, ${ }^{10}$ B. P. Crill, ${ }^{11}$ P. de Bernardis, ${ }^{12}$ G. De Gasperis, ${ }^{9}$ A. de Oliveira-Costa, ${ }^{13}$ G. De Troia, ${ }^{12}$ G. di Stefano, ${ }^{14}$ E. Hivon, ${ }^{11}$ A. H. Jaffe, ${ }^{10}$ T. S. Kisner, ${ }^{1,15}$ W. C. Jones, ${ }^{4}$ A. E. Lange, ${ }^{4}$ S. Masi, ${ }^{12}$ P. D. Mauskopf, ${ }^{2}$ C. J. MaCTavish, ${ }^{16}$ A. Melchiorri, ${ }^{12,17}$ P. Natoli, ${ }^{918}$ C. B. Netterfield, ${ }^{16,19}$ E. Pascale, ${ }^{16}$ F. Piacentini, ${ }^{12}$ D. Pogosyan, ${ }^{20}$ G. Polenta, ${ }^{12}$ S. Prunet, ${ }^{21}$ S. Ricciardi, ${ }^{12}$ G. Romeo, ${ }^{14}$ J. E. Ruhl, ${ }^{1}$ P. Santini, ${ }^{12}$ M. Tegmark, ${ }^{13}$ M. Veneziani, ${ }^{12}$ and N. Vittorio 9,18 Received 2005 July 21; accepted 2006 May 2

\begin{abstract}
We report measurements of the CMB polarization power spectra from the 2003 January Antarctic flight of BOOMERANG. The primary results come from 6 days of observation of a patch covering $0.22 \%$ of the sky centered near R.A. $=82^{\circ} .5$, decl. $=-45^{\circ}$. The observations were made using four pairs of polarization-sensitive bolometers operating in bands centered at $145 \mathrm{GHz}$. Using two independent analysis pipelines, we measure a nonzero $\langle\mathrm{EE}\rangle$ signal in the range $201<l<1000$ with a significance of $4.8 \sigma$, a $2 \sigma$ upper limit of $8.6 \mu \mathrm{K}^{2}$ for any $\langle\mathrm{BB}\rangle$ contribution, and a $2 \sigma$ upper limit of $7.0 \mu \mathrm{K}^{2}$ for the $\langle\mathrm{EB}\rangle$ spectrum. Estimates of foreground intensity fluctuations and the nondetection of $\langle\mathrm{BB}\rangle$ and $\langle\mathrm{EB}\rangle$ signals rule out any significant contribution from Galactic foregrounds. The results are consistent with a $\Lambda C D M$ cosmology seeded by adiabatic perturbations. We note that this is the first detection of $C M B$ polarization with bolometric detectors.
\end{abstract}

Subject headings: cosmic microwave background - instrumentation: detectors

Online material: color figures

\section{INTRODUCTION}

Measurements of the polarization of the cosmic microwave background (CMB) are a powerful cosmological probe. The CMB is polarized by Thomson scattering (Rees 1968) during recombination and reionization. Polarization anisotropies have an amplitude that is $\sim 10 \%$ of the temperature anisotropies (Bond $\&$ Efstathiou 1984). Similar to CMB temperature anisotropies, the angular power spectra of CMB polarization encode cosmological information. In recent years CMB temperature anisotropy measurements have provided strong constraints on fundamental cosmological parameters (see, e.g., Bond et al. 2003). The strength of these constraints relies on the assumption that initial pertur-

\footnotetext{
1 Physics Department, Case Western Reserve University, Rockefeller Building, 10900 Euclid Avenue, Cleveland, OH 44106.

2 School of Physics and Astronomy, Cardiff University, Cardiff CF24 3YB, UK.

3 Jet Propulsion Laboratory, Pasadena, CA 91109.

4 Observational Cosmology, California Institute of Technology, Pasadena, CA 91125.

5 Canadian Institute for Theoretical Astrophysics, University of Toronto, Toronto, ON M5S 3H8, Canada.

${ }^{6}$ Computational Research Division, Lawrence Berkeley National Laboratory, Berkeley, CA 94720.

7 Space Sciences Laboratory, University of California, Berkeley, CA 94720.

8 IFAC-CNR, 50127 Florence, Italy.

9 Dipartimento di Fisica, Università di Roma “Tor Vergata," 00133 Rome, Italy.

10 Theoretical Physics Group, Imperial College, London SW7 2BW, UK.

11 IPAC, California Institute of Technology, Pasadena, CA 91125.

12 Dipartimento di Fisica, Università di Roma La Sapienza, 00185 Rome, Italy.

13 Department of Physics, Massachusetts Institute of Technology, Cambridge, MA 02139.

14 Istituto Nazionale di Geofisica e Vulcanologia, 00143 Rome, Italy.

15 Department of Physics, University of California, Santa Barbara, CA 93106.

16 Physics Department, University of Toronto, Toronto, ON M5S 3H8, Canada.

17 INFN, Sezione di Roma 1, 00133 Rome, Italy.

18 INFN, Sezione di Roma 2, 00133 Rome, Italy.

19 Department of Astronomy and Astrophysics, University of Toronto, Toronto, ON M5S 3H8, Canada.

20 Physics Department, University of Alberta, Edmonton, AB T6G 2J1, Canada.

21 Institut d'Astrophysique, 75014 Paris, France.
}

bations are adiabatic in origin. If an admixture of isocurvature perturbations is allowed, then these constraints are somewhat weakened (Enqvist \& Kurki-Suonio 2000; Bucher et al. 2001). The addition of polarization information can constrain such isocurvature contributions and tighten current constraints derived from temperature anisotropies.

With increased sensitivity, future measurements of CMB polarization will provide new independent constraints on the cosmological model. A measurement of the gravitational lensing of CMB polarization could provide independent constraints on the neutrino mass, the dark energy equation of state, and the nature of reionization (Kaplinghat et al. 2003; Hu 2002). It may also be possible to obtain direct evidence of inflation through its effect on the pattern on CMB polarization (Polnarev 1985; Crittenden et al. 1993).

Any electromagnetic wave can be described by the Stokes parameters: $I$ is the intensity, $Q$ and $U$ parameterize linear polarization, and $V$ describes the circular polarization. Thomson scattering does not produce circular polarization, so we expect $V=0$ for the CMB. The parameters $Q$ and $U$ are not rotationally invariant quantities. Consequently, it is customary to characterize $\mathrm{CMB}$ polarization as the sum of curl-free and divergence-free components (Zaldarriaga \& Seljak 1997; Kamionkowski et al. 1997). Using an analogy to electromagnetism, the curl-free components are called $E$-modes and the divergence-free components are called $B$-modes. The $E$-modes and $B$-modes are related to $Q$ and $U$ by a nonlocal linear transformation. There are five observables for $\mathrm{CMB}$ polarization: the $E$-mode correlation function, $\langle\mathrm{EE}\rangle$, the $B$-mode correlation function, $\langle\mathrm{BB}\rangle$, the crosscorrelation between $E$ - and $B$-mode polarization, $\langle\mathrm{EB}\rangle$ and the cross-correlations between temperature anisotropies and polarization, $\langle\mathrm{TE}\rangle$ and $\langle\mathrm{TB}\rangle$. All of these correlations are parameterized by multipole moments $C_{l}^{X Y}$ where $\mathrm{X}$ and $\mathrm{Y}$ can represent $E$-modes, $B$-modes, or temperature anistropies.

The $E$-mode polarization of the CMB is primarily produced by scalar fluctuations on the last scattering surface, due to motion of the photon-baryon fluid, which is induced by density 
fluctuations. However, these scalar fluctuations do not produce $B$-mode polarization on the last scattering surface. Tensor perturbations induced by gravity waves can create $\mathrm{CMB}$ polarization as well. Inflationary models generically predict a spectrum of primordial gravity waves that have an amplitude proportional to the fourth power of the energy scale at the time of inflation (Turner \& White 1996). Gravity waves produce $E$ - and $B$-mode polarization in roughly equal quantities (Seljak \& Zaldarriaga 1997). Given current constraints on tensor perturbations (Seljak et al. 2005), scalar perturbations are expected to dominate the $E$-mode power spectrum by a factor of at least 10 . If parity is preserved in the early universe, then we expect there to be no correlation between $E$ - and $B$-mode polarization (i.e., $\langle\mathrm{EB}\rangle=0$ ) or between temperature anisotropies and $B$-mode polarization $(\langle\mathrm{TB}\rangle=0)$. However, it is possible to construct models in which parity is violated, and these correlations are nonzero (Pogosian et al. 2002).

In models seeded by purely adiabatic perturbations, acoustic peaks in the $E$-mode angular power spectrum should be $\sim 180^{\circ}$ out of phase with the acoustic peaks in the temperature anisotropy angular power spectrum, $\langle\mathrm{TT}\rangle$. Peaks in the $E$-mode spectrum should line up with troughs in the temperature spectrum, because the scalar component of $E$-mode polarization is related to velocities and not densities on the last scattering surface. The angular spectrum of the cross-correlation between temperature anisotropies and $E$-mode polarization $(\langle\mathrm{TE}\rangle)$ will show a series of acoustic peaks that occur between the peaks of the $\langle\mathrm{TT}\rangle$ and $\langle\mathrm{EE}\rangle$ power spectra. Measurements of the $\langle\mathrm{EE}\rangle$ power spectrum by the Degree Angular Scale Interferometer (DASI; Kovac et al. 2002; Leitch et al. 2004), Cosmic Background Imager (CBI; Readhead et al. 2004), and CAPMAP (Barkats et al. 2005) along with $\langle\mathrm{TE}\rangle$ measurements by DASI, Wilkinson Microwave Anistropy Probe (WMAP) (Kogut et al. 2003), and CBI provide evidence that the assumption of adiabatic perturbations is valid.

In this paper we report a measurement of the $E$-mode polarization power spectrum from the second Antarctic flight of BOOMERANG, which took place in 2003 January (hereafter B03). The telescope and instrument configuration from the 1997 test flight and the first Antarctic flight are discussed in Piacentini et al. (2002) and Crill et al. (2003), respectively. The instrument configuration for the 2003 flight is described in Masi et al. (2006) along with the data processing and the CMB maps. Results for the temperature anisotropy spectrum and the temperature-polarization cross-correlation are reported in Jones et al. (2006a) and Piacentini et al. (2006), respectively. Cosmological parameter constraints are reported in MacTavish et al. (2006). In this paper we briefly review the instrument and observations in $\S 2$. In $\S 3$ we discuss the analysis methods used to estimate the polarization power spectra, and in $\S 4$ we present the power spectrum results. Section 5 provides a discussion of systematic errors, and $\S 6$ describes tests for foreground contamination.

\section{INSTRUMENT AND OBSERVATIONS}

BOOMERANG is a balloon-borne telescope designed for longduration flights around Antarctica. In its first Antarctic flight (1998 December), BOOMERANG measured CMB temperature anisotropies using bolometers operating with bands centered at 90, 150, 220, and $410 \mathrm{GHz}$ (Crill et al. 2003; Ruhl et al. 2003). For the 2003 flight the receiver was redesigned to measure CMB temperature and polarization anisotropies with bands centered at 145, 245, and $345 \mathrm{GHz}$ (Masi et al. 2006). The results reported here come from four pairs of polarization-sensitive bolometers (PSBs) operating at $145 \mathrm{GHz}$ (Jones et al. 2003; Jones et al. 2006b).

The 2003 flight of BOOMERANG was launched on 2003 January 6 from McMurdo Station (Antarctica) and lasted 14 days.
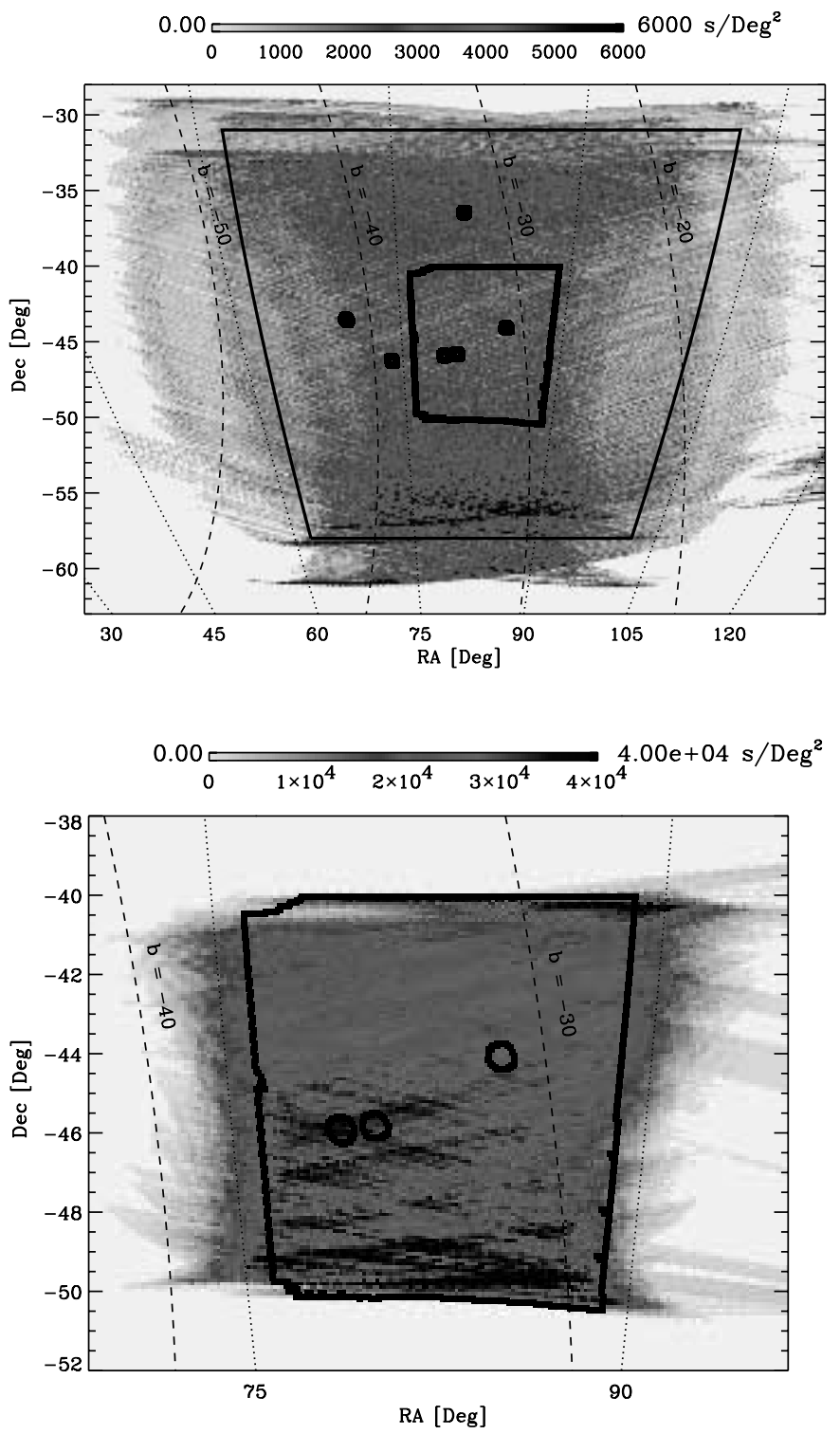

FIG. 1.-Sky coverage from the 2003 BOOMERANG flight. The top panel shows the large region covered during the first part of the flight (the shallow region), and the bottom panel is the smaller region covered during the second half of the flight (the deep region). In the top panel the outer set of black lines shows the sky cut used in the shallow mask. The inner outline shows the outline of the deep region sky cut. The shallow scans covered $3.0 \%$ of the sky, and sky cut used for the CMB analysis covers $1.8 \%$. The deep region observations covered $0.28 \%$ of the sky, and the outlined region covering $0.22 \%$ of the sky was used for the CMB analysis. The integration time per pixel for the deep observations is roughly 20 times longer than the integration time per pixel during shallow observations. In both panels, the small circles represent regions of map that are excised due to the presence of known point sources. [See the electronic edition of the Journal for a color version of this figure.]

In this paper we report on the analysis of $205 \mathrm{hr}$ of CMB observations during the first 11 days. During this period, $75 \mathrm{hr}$ were spent scanning a large region (called the shallow region) comprising $3.0 \%$ of the sky and $125 \mathrm{hr}$ on a small region (called the deep region) comprising $0.28 \%$ of the sky. The shallow region was designed to optimally measure the degree scale signals in $\langle\mathrm{TT}\rangle$ and $\langle\mathrm{TE}\rangle$, while the deep region was designed to optimize the signal-to-noise ratio on the $\langle\mathrm{EE}\rangle,\langle\mathrm{BB}\rangle$, and $\langle\mathrm{EB}\rangle$ power spectra. For these spectra almost all the statistical weight comes from the deep region.

Figure 1 shows the distribution of integration time over the observation region and the sky cuts used in the analysis. The time per pixel in the deep region is a factor of $\sim 20$ longer than in the 
shallow region. For the spectral analysis, we use $1.8 \%$ of the sky for the shallow region and $0.22 \%$ of the sky for the deep region. These choices were made so that the coverage was roughly uniform in time per sky pixel and for the different channels in the focal plane. Another consideration was that the deep and shallow observations could each be split in half (in time) and still cover their respective sky cuts (which is useful for systematic tests).

\section{DATA ANALYSIS METHODS}

We used two independent pipelines for the map-making and polarization power spectrum estimation. Masi et al. (2006) describes the bulk of the data analysis from raw data to CMB maps including raw data cleaning, detector characterization, pointing reconstruction, calibration, beam measurement, noise estimation, and making polarized maps. In this paper we limit the discussion to aspects of power spectrum estimation for polarized data sets.

The two pipelines are independent to a high degree. In the following "NA pipeline" refers to the pipeline primarily based in North America, and "IT pipeline" refers to the pipeline developed in Italy. Each team had many choices to make about instrument characteristics and data analysis techniques. The boundaries of the shallow and deep region sky cuts used by both teams are identical, but differences in the data cleaning causes slight differences in integration time. As we show in $\S 4$, the two pipelines yield compatible answers; this is a testament to the robustness of the data set.

\subsection{Power Spectrum Estimation}

Both pipelines are polarized extensions of the Monte Carlobased MASTER method (Hivon et al. 2002) first used on B98 (Netterfield et al. 2002). These techniques rely on spherical harmonic transformations done on a partial map of the sky. For polarization data the $Q$ and $U$ maps are expanded as a function of spin-2 spherical harmonics

$$
(Q \pm i U)(\hat{\boldsymbol{n}})=\sum_{l m}\left(a_{l m}^{\mathrm{E}} \pm i a_{l m}^{\mathrm{B}}\right)_{ \pm 2} Y_{l m}(\hat{\boldsymbol{n}}),
$$

where $a_{l m}^{\mathrm{E}}$ and $a_{l m}^{\mathrm{B}}$ are the coefficients for $E$ - and $B$-mode polarization, respectively (Zaldarriaga \& Seljak 1997; Kamionkowski et al. 1997). These coefficients can be calculated in a manner similar to Legendre transformations,

$$
\begin{aligned}
a_{l m}^{E}=\frac{1}{2} \int d \Omega W(\hat{\boldsymbol{n}})[ & (Q+i U)(\hat{\boldsymbol{n}})_{+2} Y_{l m}(\hat{\boldsymbol{n}}) \\
& \left.+(Q-i U)(\hat{\boldsymbol{n}})_{-2} Y_{l m}(\hat{\boldsymbol{n}})\right], \\
a_{l m}^{B}=\frac{1}{2 i} \int d \Omega W(\hat{\boldsymbol{n}})[ & (Q+i U)(\hat{\boldsymbol{n}})_{+2} Y_{l m}(\hat{\boldsymbol{n}}) \\
& \left.-(Q-i U)(\hat{\boldsymbol{n}})_{-2} Y_{l m}(\hat{\boldsymbol{n}})\right],
\end{aligned}
$$

where $W(\hat{\boldsymbol{n}})$ is an arbitrary weighting function and the integral extends only over the observed portion of the sky. From these transforms we can build three observables,

$$
\begin{aligned}
C_{l}^{\mathrm{EE}} & =\frac{1}{2 l+1} \sum_{m=-l}^{l}\left|a_{l m}^{\mathrm{E}}\right|^{2}, \\
C_{l}^{\mathrm{BB}} & =\frac{1}{2 l+1} \sum_{m=-l}^{l}\left|a_{l m}^{\mathrm{B}}\right|^{2}, \\
C_{l}^{\mathrm{EB}} & =\frac{1}{2 l+1} \sum_{m=-l}^{l} a_{l m}^{\mathrm{E}} a_{l m}^{\mathrm{B} *},
\end{aligned}
$$

where $C_{l}^{\mathrm{EE}}$ is the $E$-mode power spectrum, $C_{l}^{\mathrm{BB}}$ is the $B$-mode power spectrum, and $C_{I}^{\mathrm{EB}}$ is the cross-correlation between $E$ - and $B$-mode polarization. The spectrum $C_{l}^{\mathrm{EB}}$ is expected to be zero if parity is preserved in the early universe. Our estimates of the cross-correlations between temperature and polarization $\left(C_{l}^{\mathrm{TE}}\right.$ and $C_{l}^{\mathrm{TB}}$ ) are discussed in Piacentini et al. (2006).

For spherical harmonic transforms done on the cut sky the measure of $C_{l}$ is biased; we describe them as pseudo- $C_{l}$ terms $\left(\tilde{C}_{l}\right)$. For the polarization power spectra, the relationships between full-sky $C_{l}$ and $\tilde{C}_{l}$ are expressed as

$$
\begin{aligned}
& \tilde{C}_{l}^{\mathrm{EE}}=\sum_{l^{\prime}}\left({ }_{+} K_{l l^{\prime}} F_{l^{\prime}}^{\mathrm{EE}} B_{l^{\prime}}^{2} C_{l^{\prime}}^{\mathrm{EE}}+{ }_{-} K_{l l^{\prime}} F_{l^{\prime}}^{\mathrm{BB}} B_{l^{\prime}}^{2} C_{l^{\prime}}^{\mathrm{BB}}\right)+\tilde{N}_{l}^{\mathrm{EE}}, \\
& \tilde{C}_{l}^{\mathrm{BB}}=\sum_{l^{\prime}}\left({ }_{+} K_{l l^{\prime}} F_{l^{\prime}}^{\mathrm{BB}} B_{l^{\prime}}^{2} C_{l^{\prime}}^{\mathrm{BB}}+{ }_{-} K_{l l^{\prime}} F_{l^{\prime}}^{\mathrm{EE}} B_{l^{\prime}}^{2} C_{l^{\prime}}^{\mathrm{EE}}\right)+\tilde{N}_{l}^{\mathrm{BB},} \\
& \tilde{C}_{l}^{\mathrm{EB}}=\sum_{l^{\prime}}\left({ }_{+} K_{l l^{\prime}}-{ }_{-} K_{l l^{\prime}}\right) F_{l^{\prime}}^{\mathrm{EB}} B_{l^{\prime}}^{2} C_{l^{\prime}}^{\mathrm{EB}}+\tilde{N}_{l}^{\mathrm{EB}},
\end{aligned}
$$

where $C_{l}^{\mathrm{XY}}$ represents the full-sky power spectrum, $B_{l}$ is the beam window function, $F_{l}^{\mathrm{XY}}$ is the transfer function measured by signalonly Monte Carlo simulations, $\tilde{N}_{l}^{\mathrm{XY}}$ is the noise bias measured by noise-only Monte Carlo simulations, ${ }_{+} K_{l l^{\prime}}$ is the primary coupling kernel, and $K_{l l^{\prime}}$ describes the geometric leakage between $E$ - and $B$-modes (Chon et al. 2004). Having computed the pseudo- $C_{l}$ terms, there are two conventional methods to estimate the full-sky power spectra. Equations (7), (8), and (9) are linear in $C_{l}^{\mathrm{XY}}$, but the coupling kernels are singular when the spherical harmonic transformations are done on the cut sky. It is possible to solve for $C_{l}^{\mathrm{XY}}$ by binning the above equations in $l$ (see, e.g., Hivon et al. 2002; Kogut et al. 2003; Challinor \& Chon 2005). The spectrum $C_{l}^{\mathrm{XY}}$ can also be estimated using quadratic estimator techniques (Bond et al. 2000).

Both pipelines use roughly 500 Monte Carlo simulations of signal- and noise-only data streams to estimate the signal transfer function and noise bias, respectively. A similar number of signal+noise simulations can be used to estimate the uncertainty on the spectral estimate and check for bias in the pipeline.

Since we observe a small portion of the sky, we are not able to measure individual multipole moments. Instead, we parameterize the power spectrum as a piecewise continuous function,

$$
C_{l}^{\mathrm{XY}}=q_{b}^{\mathrm{XY}} C_{l}^{(S) \mathrm{XY}},
$$

where $q_{b}^{\mathrm{XY}}$ is the band power deviation over a range $(\Delta l)_{b}$ and $C_{l}^{(S) \mathrm{XY}}$ is a shape parameter. Common choices for the shape parameter are those that keep $C_{l}^{(S)}$ constant over the band, those that keep $l(l+1) C_{l}^{(S)} /(2 \pi)$ constant over the band (i.e., the flattened spectrum), or those that represent a theoretically motivated power spectrum (e.g., the $\Lambda \mathrm{CDM}$ concordance model). The choice of parameterization depends in part on the nature of the expected signal and the noise in the maps. The band power deviations, $q_{b}$, are related to the average power in the band,

$$
\mathcal{C}_{b}=q_{b} \frac{\mathcal{I}\left(W_{l}^{b} \mathcal{C}_{l}^{(S)}\right)}{\mathcal{I}\left(W_{l}^{b}\right)},
$$

where $\mathcal{C}_{l}^{(S)}=l(l+1) C_{l}^{(S)} / 2 \pi, W_{l}^{b}$ is the band power window function (Knox 1999), and $\mathcal{I}\left(\boldsymbol{f}_{l}\right)$ is the logarithmic integral (Bond et al. 2000),

$$
\mathcal{I}\left(f_{l}\right)=\sum_{l} \frac{l+1 / 2}{l(l+1)} f_{l}
$$


Using this formalism, we can calculate the expected band power deviation for a given theoretical model

$$
\left\langle q_{b}\right\rangle=\frac{\mathcal{I}\left(W_{l}^{b} \mathcal{C}_{l}\right)}{\mathcal{I}\left(W_{l}^{b} \mathcal{C}_{l}^{(S)}\right)}
$$

\subsection{NA Pipeline}

For the NA pipeline, a quadratic estimator (Bond et al. 1998) is used to iteratively solve for the band powers and their uncertainty. This estimator (called Xfaster) is capable of solving for the power spectra of a single map or any combination of two or more maps (which can be overlapping) while accounting for all correlations between those maps (C. R. Contaldi et al. 2006, in preparation).

For B03 Xfaster is used to solve for the combined power spectrum of the shallow and deep region data (the combined power spectra are called the 2Mask spectra). Separate maps are made from the shallow and deep region observations; this ensures that the only correlations between the maps are due to sky signal. When we perform the spherical harmonic transformations, we use a uniform pixel weighting for the shallow map, and pixels in the deep region are weighted by the inverse square root of noise in that pixel $\left(\sigma_{\text {pix }}^{-1 / 2}\right)$. An effective noise weighting is also applied in the spectrum estimation process due to noise bias of each map $\left(\tilde{N}_{l}^{\mathrm{XY}}\right)$. This is an efficient way to account for the imbalance of integration time per pixel between the shallow and deep maps.

Although the shallow region does not contribute much statistical weight to the polarization spectra, it does significantly reduce the sample variance in the $\langle\mathrm{TT}\rangle$ and $\langle\mathrm{TE}\rangle$ spectra. The Xfaster method is used for the polarization spectra so that we can derive consistent correlation matrices between all spectra for use in parameter estimation (MacTavish et al. 2006).

\subsection{IT Pipeline}

To solve for the power spectrum, the IT pipeline uses a method similar to that described in Hivon et al. (2002) but adapted for polarization spectra (see, e.g., Kogut et al. 2003 and Challinor \& Chon 2005). Errors bars and correlation matrices are calculated using band powers resulting from signal+noise Monte Carlo simulations. The IT pipeline performs the $\langle\mathrm{EE}\rangle,\langle\mathrm{BB}\rangle$, and $\langle\mathrm{EB}\rangle$ analysis on the deep region maps with the pixels weighted by the inverse square root of the noise $\left(\sigma_{\mathrm{pix}}^{-1 / 2}\right)$. The IT maps also include data from shallow observations that fall inside the deep region.

\subsection{Testing Goodness of Fit}

To test how well particular models fit our data, we use the standard likelihood ratio technique in a manner similar to recent work done by DASI (Kovac et al. 2002; Leitch et al. 2004) and CBI (Readhead et al. 2004). Specifically, we calculate the logarithm of the ratio of the peak of the likelihood to the likelihood of a model $M$ parameterized by band powers $\mathcal{C}_{b}$,

$$
\Lambda\left(\mathcal{C}_{b}\right)=\ln \left[\frac{L\left(\overline{\mathcal{C}}_{b}\right)}{L\left(\mathcal{C}_{b}\right)}\right]
$$

where $\overline{\mathcal{C}}_{b}$ are the maximum-likelihood band powers. The larger this ratio is, the worse the model $M$ fits the data. In this paper we are primarily concerned with comparisons to the null hypothesis $\left(\mathcal{C}_{b}=0\right.$ for all $\left.b\right)$ and our fiducial $\Lambda \mathrm{CDM}$ model (the best fit to the $W M A P\langle\mathrm{TT}\rangle$ spectra from Spergel et al. 2003).

In the approximation that the likelihood function $L\left(\mathcal{C}_{b}\right)$ is a multivariate Gaussian near its peak we have $\Lambda=\Delta \chi^{2} / 2$. This is generally a good approximation when the signal-to-noise ratio in a band is low, and there is a large number of modes in the band $(l>50)$. We confirmed this assumption with Monte Carlo simulations.

If we assume that a given model $M$ is true, the probability of observing $\Lambda$ exceeding a particular value is given by the "probability to exceed" (PTE),

$$
\operatorname{PTE}(\Lambda)=\frac{1}{\Gamma(N / 2)} \int_{\Lambda}^{\infty} e^{-x} x^{(N / 2)-1} d x
$$

where $N$ is the number of parameters and $\Gamma(x)$ is the complete gamma function. For example, if PTE $=5 \%$, then we can reject the hypothesis that the model $M$ is true with $95 \%$ confidence. Given that we have only used 500 simulations to confirm the Gaussianity of the error bars, we do not have enough statistics to confirm that any PTE $<0.01$ is accurate. Low values of PTE should be considered strictly formal and are intended to be used to compare results from different experiments.

Since our power spectrum estimators do not calculate full likelihood values, we use the offset log-normal function, $Z_{b}=$ $\ln \left(\mathcal{C}_{b}+x_{b}\right)$, to approximate the likelihood function (Bond et al. $2000)$, where $\mathcal{C}_{b}$ is the band power and $x_{b}$ is the offset parameter. The likelihood is calculated by

$$
\begin{aligned}
\sigma_{b} & =\Delta \overline{\mathcal{C}}_{b} /\left(\overline{\mathcal{C}}_{b}+x_{b}\right), \\
\Delta Z_{b} & =\ln \left(\mathcal{C}_{b}+x_{b}\right)-\ln \left(\overline{\mathcal{C}}_{b}+x_{b}\right), \\
-2 \ln L\left(\mathcal{C}_{b}\right) & =\sum_{b b^{\prime}} \Delta Z_{b} \sigma_{b}^{-1} G_{b b^{\prime}} \sigma_{b^{\prime}}^{-1} \Delta Z_{b^{\prime}}^{-1},
\end{aligned}
$$

where $\overline{\mathcal{C}}_{b}$ is the maximum-likelihood band power and $G_{b b^{\prime}}$ is the band power correlation matrix, which is normalized to unity on the diagonals. In this parameterization, the likelihood is normalized to the peak value [i.e., $\ln L\left(\overline{\mathcal{C}}_{b}\right)=0$ ].

In the results to follow, we compute $\Lambda$ and PTE separately for the $\langle\mathrm{EE}\rangle,\langle\mathrm{BB}\rangle$, and $\langle\mathrm{EB}\rangle$ spectra. In other words, when performing this test on one spectrum, we marginalize over the other spectra by excising the correlations between spectra from the inverse Fisher matrix.

\section{RESULTS}

\subsection{Narrowband Analysis}

The $\langle\mathrm{EE}\rangle,\langle\mathrm{BB}\rangle$, and $\langle\mathrm{EB}\rangle$ power spectra results from both pipelines are shown in Figure 2 and listed in Table 1. The multipole range shown is from $101 \leq l \leq 1100$; information on scales $2 \leq l \leq 100$ and $l \geq 1101$ is discarded (spin-2 spherical harmonics do not exist for $l<2$ ). These data, $x_{b}$ values, window functions, and correlation matrices are available from the instrument Web sites. ${ }^{22}$

Although we use a shape function that is constant in $l(l+1) C_{l} /$ $(2 \pi)$, we choose to plot the results in terms of $(2 l+1) C_{l} /(4 \pi)$ to emphasize our sensitivity in the range $100<l<1100$ and relate the power spectrum directly to the rms CMB signal,

$$
\sigma_{\mathrm{CMB}}^{2}=\sum_{l} \frac{2 l+1}{4 \pi} C_{l} .
$$

The results from the two pipelines agree rather well, but there is some scatter in the $\langle\mathrm{EE}\rangle$ spectrum results. The power spectrum results are very sensitive to the process of detector noise

\footnotetext{
${ }^{22}$ See http://cmb.case.edu/boomerang and http://oberon.roma1.infn.it/ boomerang.
} 


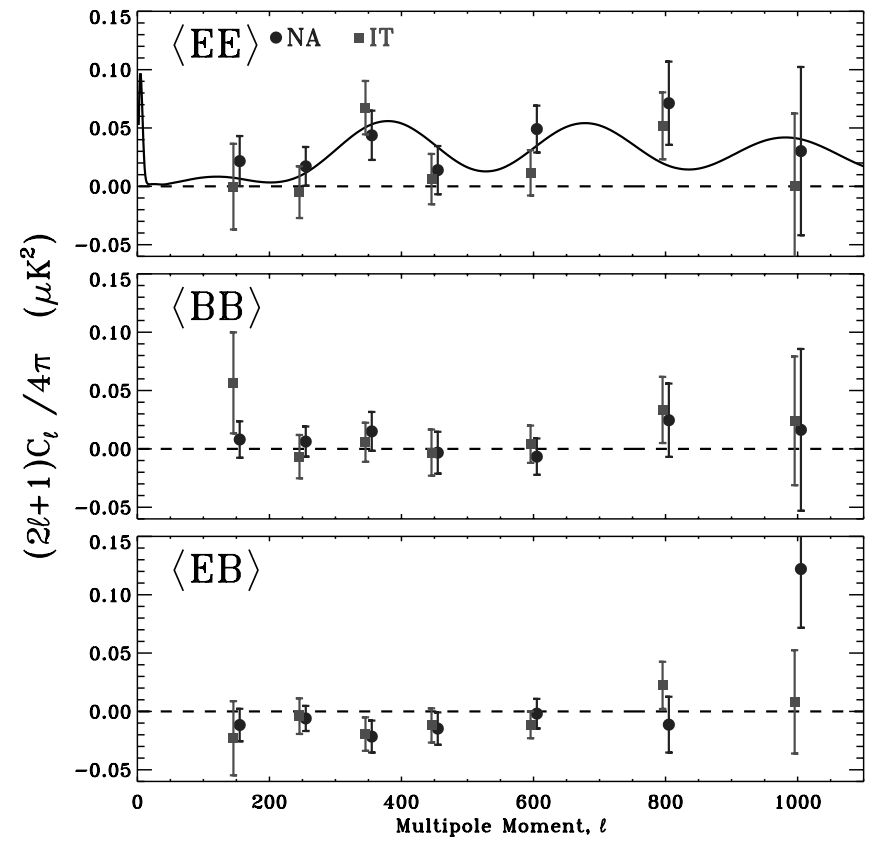

Fig. 2.-Angular power spectra results. From top to bottom the panels show the $\langle\mathrm{EE}\rangle,\langle\mathrm{BB}\rangle$, and $\langle\mathrm{EB}\rangle$ power spectrum results from the NA (circles) and IT (squares) pipelines. The solid line in the $\langle\mathrm{EE}\rangle$ plot is the best-fit $\Lambda \mathrm{CDM}$ model to the WMAP $\langle\mathrm{TT}\rangle$ results (Spergel et al. 2003), and the dashed line in all plots represent zero-signal. From these plots and the statistical tests in Table 2, it is clear the $\langle\mathrm{BB}\rangle$ and $\langle\mathrm{EB}\rangle$ are consistent with zero signal while $\langle\mathrm{EE}\rangle$ is consistent with $\Lambda \mathrm{CDM}$, but inconsistent with zero signal. The $\Lambda \mathrm{CDM}\langle\mathrm{EE}\rangle$ model predicted by the WMAP $\langle\mathrm{TT}\rangle$ results is nearly identical to the best-fit $\langle\mathrm{EE}\rangle$ model predicted from recent $\langle\mathrm{TT}\rangle$ results including B03 (MacTavish et al. 2006). [See the electronic edition of the Journal for a color version of this figure.]

estimation; misestimation of the noise in the map leads directly to a bias in the power spectrum estimate. The IT results assumed that the noise properties of each detector were constant throughout the flight, while the NA results assumed that the noise properties were constant on $1 \mathrm{hr}$ timescales. As discussed in Masi et al. (2006), variations in the payload altitude lead to changes in the detector responsivity. This in turn leads to changes of a few percent in the detector noise properties. The altitude variations were slow enough that the assumption of stationary noise on $1 \mathrm{hr}$ timescales is a reasonable approximation. Simulations done with the NA pipeline show that the assumption of stationary noise over the whole flight can lead to disagreements similar in size to the disagreements between the NA and IT results shown here. For this reason, we consider the NA results to be more robust.

In Figure 2 only the $\langle E E\rangle$ spectrum appears to be significantly different from zero. To quantify this, we calculate the $\Lambda$ statistic for the assumption of zero polarized signal. The results in Table 2 show that the $\langle E E\rangle$ result is inconsistent with zero signal and that $\langle\mathrm{BB}\rangle$ and $\langle\mathrm{EB}\rangle$ are both consistent with zero. Similarly, we compare $\langle\mathrm{EE}\rangle$ and $\langle\mathrm{BB}\rangle$ to the $E$-mode power spectrum from the fiducial $\Lambda \mathrm{CDM}$ model. In this case the $\langle\mathrm{EE}\rangle$ result is in good agreement with the fiducial $E$-mode spectrum, while $\langle\mathrm{BB}\rangle$ is not. For these calculations the IT data and the NA $\langle\mathrm{EB}\rangle$ data are taken to have $x_{b}=0$.

\subsection{Wideband Analysis}

To assess the raw significance of our $\langle\mathrm{EE}\rangle$ result and to set an upper limit for $\langle\mathrm{BB}\rangle$ and $\langle\mathrm{EB}\rangle$ spectra, we perform a wideband analysis over a range $201 \leq l \leq 1000$ (the actual analysis uses three bins with bins defined by $2 \leq l \leq 200$ and $1001 \leq l \leq 1999$
TABLE 1

Power Spectra Results for $\langle\mathrm{EE}\rangle,\langle\mathrm{BB}\rangle$, and $\langle\mathrm{EB}\rangle$ from the NA and IT ANalysis Pipelines

\begin{tabular}{|c|c|c|c|c|c|c|}
\hline \multirow[b]{2}{*}{$l_{\min }$} & \multirow[b]{2}{*}{$l_{\max }$} & \multirow[b]{2}{*}{$l_{\text {center }}$} & \multicolumn{2}{|c|}{ NA } & \multicolumn{2}{|c|}{ IT } \\
\hline & & & $\begin{array}{c}\overline{\mathcal{C}}_{b} \\
\left(\mu \mathrm{K}^{2}\right)\end{array}$ & $\begin{array}{c}\Delta \overline{\mathcal{C}}_{b} \\
\left(\mu \mathrm{K}^{2}\right)\end{array}$ & $\begin{array}{c}\overline{\mathcal{C}}_{b} \\
\left(\mu \mathrm{K}^{2}\right)\end{array}$ & $\begin{array}{c}\Delta \overline{\mathcal{C}}_{b} \\
\left(\mu \mathrm{K}^{2}\right)\end{array}$ \\
\hline \multicolumn{7}{|c|}{$\langle\mathrm{EE}\rangle$} \\
\hline 101 & 200 & 150 & 3.3 & 3.2 & -0.04 & 5.5 \\
\hline 201 & 300 & 250 & 4.3 & 4.2 & -1.24 & 5.6 \\
\hline 301 & 400 & 350 & 15.3 & 7.40 & 23.7 & 8.0 \\
\hline 401 & 500 & 450 & 6.3 & 9.3 & 2.8 & 9.7 \\
\hline 501 & 700 & 600 & 29.5 & 12.1 & 6.94 & 11.7 \\
\hline 701 & 900 & 800 & 57.0 & 28.6 & 41.6 & 23.0 \\
\hline 901 & 1100 & 1000 & 30.2 & 72.1 & 0.3 & 62.2 \\
\hline \multicolumn{7}{|c|}{$\langle\mathrm{BB}\rangle$} \\
\hline 101 & 200 & 150 & 1.2 & 2.4 & 8.5 & 6.5 \\
\hline 201 & 300 & 250 & 1.6 & 3.2 & -1.7 & 4.7 \\
\hline 301 & 400 & 350 & 5.3 & 5.8 & 2.0 & 5.9 \\
\hline 401 & 500 & 450 & -1.5 & 8.1 & -1.4 & 8.9 \\
\hline 501 & 700 & 600 & -4.0 & 9.3 & 2.4 & 9.6 \\
\hline 701 & 900 & 800 & 19.7 & 25.2 & 26.7 & 22.7 \\
\hline 901 & 1100 & 1000 & 16.3 & 69.4 & 24.1 & 55.2 \\
\hline \multicolumn{7}{|c|}{$\langle\mathrm{EB}\rangle$} \\
\hline 101 & 200 & 150 & -1.8 & 2.1 & -3.5 & 4.8 \\
\hline 201 & 300 & 250 & -1.5 & 2.7 & -1.0 & 3.8 \\
\hline 301 & 400 & 350 & -7.6 & 4.8 & -6.8 & 5.0 \\
\hline 401 & 500 & 450 & -6.6 & 6.3 & -5.4 & 6.6 \\
\hline 501 & 700 & 600 & -1.1 & 7.6 & -6.9 & 6.9 \\
\hline 701 & 900 & 800 & -9.1 & 19.1 & 17.9 & 16.2 \\
\hline 901 & 1100 & 1100 & 122.1 & 50.4 & 8.2 & 44.3 \\
\hline
\end{tabular}

Notes.-The first three columns define the $l$-bins used, and the next four columns state the band powers and errors from both pipelines, $\overline{\mathcal{C}}_{b}$ and $\Delta \overline{\mathcal{C}}_{b}\left(\mathcal{C}_{l}=\right.$ $l(l+1) C_{l} / 2 \pi$, respectively. Power spectra, $x_{b}$ 's, window functions, and correlation matrices are available at http://cmb.case.edu/boomerang and http://oberon .romal.infn.it/boomerang.

used as "junk" bins). We used four different shape functions for the band powers: constant in $C_{l}$, constant in $(2 l+1) C_{l} / 4 \pi$, constant in $l(l+1) C_{l} / 2 \pi$, and the fiducial $\Lambda \mathrm{CDM}$ model. Sky cuts and spectrum estimation are the same as those used in the narrowband analysis.

Table 3 shows the band power results for all cases of the wideband analysis, and Table 4 shows the $\Lambda$ statistic and PTE for each case. In all cases the $\langle\mathrm{BB}\rangle$ and $\langle\mathrm{EB}\rangle$ results are consistent with zero signal, while the $\langle\mathrm{EE}\rangle$ signal is significantly nonzero. The choice of shape function and the fine details of the band power estimator have an effect on the output band powers. The NA and IT band powers agree closely with the results from Monte Carlo simulations of each method using the fiducial $\Lambda \mathrm{CDM}$ model as the input. When we parameterize the spectra as flat in $l(l+1) C_{l} /(2 \pi)$, the simulations also show that the difference in error bars is consistent with the differences between the NA and IT estimators. This difference is illustrated by the top panel of Figure 3, which shows the wideband window function used by the NA Xfaster estimator. Xfaster performs an effective Wiener filter, in which most of the statistical weight comes from the lower edge of the band. The IT estimator uses a flat window function, which leads to a less optimal result. The bottom panel of Figure 3 shows how the NA window functions vary with the choice of shape function. 
TABLE 2

Values for the $\Lambda$ Statistic and PTE Calculated from the Narrowband Results

\begin{tabular}{|c|c|c|c|c|c|c|c|c|}
\hline \multirow[b]{3}{*}{ Observables } & \multicolumn{4}{|c|}{ Fiducial Model } & \multicolumn{4}{|c|}{ No Polarization } \\
\hline & \multicolumn{2}{|c|}{ NA } & \multicolumn{2}{|c|}{ IT } & \multicolumn{2}{|c|}{ NA } & \multicolumn{2}{|c|}{ IT } \\
\hline & $\Lambda$ & PTE & $\Lambda$ & PTE & $\Lambda$ & PTE & $\Lambda$ & PTE \\
\hline$\langle\mathrm{EE}\rangle \ldots \ldots \ldots$ & 2.6 & 0.63 & 3.2 & 0.49 & 11.8 & $1.3 \times 10^{-3}$ & 7.2 & 0.05 \\
\hline$\langle\mathrm{BB}\rangle$ & 6.6 & 0.07 & 9.2 & 0.01 & 1.3 & 0.92 & 2.0 & 0.78 \\
\hline$\langle\mathrm{EB}\rangle$ & $\ldots$ & $\ldots$ & $\ldots$ & $\ldots$ & 5.8 & 0.11 & 2.6 & 0.64 \\
\hline
\end{tabular}

Notes.-Calculated from the narrowband results in Fig. 2 and Table 1. We compare $\langle\mathrm{EE}\rangle$ and $\langle\mathrm{BB}\rangle$ to the fiducial $\Lambda \mathrm{CDM}$ model (the best fit to the $W M A P\langle\mathrm{TT}\rangle$ from Spergel et al. 2003), and we test the null hypothesis (no polarization signal) on all three spectra. Both data sets are assumed to have 7 degrees of freedom. For both data sets $\langle\mathrm{EE}\rangle$ is a good fit to the fiducial model and inconsistent with the null hypothesis. $\langle\mathrm{BB}\rangle$ is not a good fit to the fiducial model but is consistent with no signal. $\langle\mathrm{EB}\rangle$ is consistent with zero signal.

The use of different power spectrum estimation techniques also explains the differences between the NA and IT wideband $\langle\mathrm{EB}\rangle$ results. As shown in Figure 2, the $\langle\mathrm{EB}\rangle$ narrowband spectra agree well for $l<500$. The $\langle\mathrm{EB}\rangle$ power spectrum is relatively immune to noise bias, so we expect the spectra to agree well for the different pipelines. For the NA results the Xfaster technique derives most of its statistical power from $l<600$, while the IT estimator has an equal weight over the band. The extra weight given to the data at higher $l$ brings the IT band power closer to zero.

For each parameterization the $\Lambda$ statistic and PTE results from the wideband analysis are reported in Table 4 . For a single band power the significance of the detection can be calculated by

$$
S=\sqrt{2 \Lambda}
$$

where $S$ is the detection significance quoted in units of $\sigma$. With the NA results we find that the shape function that is constant in $C_{l} / 2 \pi$ produces the highest significance, but its significance is only slightly higher than what we find when using $(2 l+1) C_{l} / 4 \pi$ or $\Lambda \mathrm{CDM}$ as the shape function. For the case parameterized as constant in $C_{l}$, we find that the $\langle\mathrm{EE}\rangle$ band power is consistent with a $4.8 \sigma$ detection. For this same case we quote $2 \sigma$ upper limits of $8.6 \mu \mathrm{K}^{2}$ for $\langle\mathrm{BB}\rangle$ and $7.0 \mu \mathrm{K}^{2}$ for $\langle\mathrm{EB}\rangle$.

\section{SYSTEMATIC ERRORS}

Given the small amplitude of the polarization signal, we need tight control on systematic errors. For most systematic errors, we would expect them to contribute equally to $\langle\mathrm{EE}\rangle$ and $\langle\mathrm{BB}\rangle$, which could also lead to a nonzero $\langle\mathrm{EB}\rangle$. The fact that $\langle\mathrm{BB}\rangle$ and $\langle\mathrm{EB}\rangle$ are consistent with zero gives credibility to the $\langle\mathrm{EE}\rangle$ result. To further establish the robustness of our result, we performed two types of internal consistency checks and a suite of Monte Carlo simulations to determine limits on systematic errors due to instrument mischaracterization.

\subsection{Internal Consistency Tests}

To check the consistency of our result, we performed jackknife tests, which are done by splitting the data in half, making maps $\Delta_{1}$ and $\Delta_{2}$ from each half and measuring the power spectrum of $\left(\Delta_{1}-\Delta_{2}\right) / 2$. If this power spectrum is consistent with zero, then the data set is considered to be internally consistent. We performed two sets of jackknife tests. The first test involves splitting the data in time [called the $(h 1-h 2) / 2$ test]. The second test is done by comparing detectors on the left and right side of the focal plane [called the $(\mathrm{WX}-\mathrm{YZ}) / 2$ test]. The $(h 1-h 2) / 2$ is sensitive to time-varying systematic problems, while the (WX-YZ)/2 is sensitive to problems affecting individual channels.

The mapmaking process for the $(h 1-h 2) / 2$ test is different in the NA and IT pipelines. With the NA pipeline, we make maps from the first and second half of the shallow observations $\left(\Delta_{h 1}^{S}\right.$ and $\Delta_{h 2}^{S}$ ), and first and second half of the deep observations $\left(\Delta_{h 1}^{D}\right.$ and $\left.\Delta_{h 2}^{D}\right)$. We then use Xfaster to estimate the combined power spectrum of $\left(\Delta_{h 1}^{S}-\Delta_{h 2}^{S}\right) / 2$ and $\left(\Delta_{h 1}^{D}-\Delta_{h 2}^{D}\right) / 2$. With the IT pipeline, we make a combined map from the first half of the shallow observations and the first half of deep observations $\Delta_{h 1}^{S D}$. Similarly, $\Delta_{h 2}^{S D}$ is made from second halves of the shallow and deep observations. The IT estimator is then used to estimate the power spectrum of $\left(\Delta_{h 1}^{S D}-\Delta_{h 2}^{S D}\right) / 2$ on the deep region mask.

TABLE 3

Wideband Analysis Results for $201 \leq l \leq 1000$

\begin{tabular}{|c|c|c|c|c|c|c|c|c|}
\hline \multirow[b]{2}{*}{$\begin{array}{c}\text { Shape } \\
\text { (1) }\end{array}$} & \multirow[b]{2}{*}{$\begin{array}{l}\text { PIPELINE } \\
\text { (2) }\end{array}$} & \multirow[b]{2}{*}{$\begin{array}{c}\left\langle\mathcal{C}_{b}^{\mathrm{EE}}\right\rangle \\
\left(\mu \mathrm{K}^{2}\right) \\
(3)\end{array}$} & \multicolumn{2}{|c|}{$\langle\mathrm{EE}\rangle$} & \multicolumn{2}{|c|}{$\langle\mathrm{BB}\rangle$} & \multicolumn{2}{|c|}{$\langle\mathrm{EB}\rangle$} \\
\hline & & & $\begin{array}{c}\mathcal{C}_{b} \\
\left(\mu \mathrm{K}^{2}\right) \\
(4)\end{array}$ & $\begin{array}{c}\Delta \mathcal{C}_{b} \\
\left(\mu \mathrm{K}^{2}\right) \\
(5)\end{array}$ & $\begin{array}{c}\mathcal{C}_{b} \\
\left(\mu \mathrm{K}^{2}\right) \\
(6)\end{array}$ & $\begin{array}{c}\Delta \mathcal{C}_{b} \\
\left(\mu \mathrm{K}^{2}\right) \\
(7)\end{array}$ & $\begin{array}{c}\mathcal{C}_{b} \\
\left(\mu \mathrm{K}^{2}\right) \\
(8)\end{array}$ & $\begin{array}{c}\Delta \mathcal{C}_{b} \\
\left(\mu \mathrm{K}^{2}\right) \\
(9)\end{array}$ \\
\hline \multirow[t]{2}{*}{$l(l+1) C_{l} /(2 \pi) \ldots \ldots$} & NA 2mask & 9.94 & 11.5 & 3.0 & 2.0 & 2.2 & -4.00 & 1.9 \\
\hline & IT deep & 15.5 & 14.2 & 6.3 & 8.0 & 5.7 & -0.2 & 3.9 \\
\hline$C_{l} \ldots \ldots \ldots+\ldots$ & NA 2 mask & 19.4 & 23.4 & 5.2 & 3.3 & 4.3 & -4.7 & 3.5 \\
\hline$(2 l+1) C_{l} / 4 \pi \ldots \ldots \ldots \ldots \ldots \ldots \ldots \ldots$ & NA 2 mask & 14.9 & 17.5 & 4.0 & 2.5 & 3.15 & -5.1 & 2.6 \\
\hline$\Lambda \mathrm{CDM}$ & NA 2mask & 15.4 & 16.4 & 3.8 & 3.3 & 3.0 & $\ldots$ & $\ldots$ \\
\hline
\end{tabular}

Notes. - The shape column refers to the band power parameterization: flat in $l(l+1) C_{l} /(2 \pi)$, flat in $C_{l}$, and $\Lambda \mathrm{CDM}$. The column labeled $\left\langle\mathcal{C}_{b}^{\mathrm{EE}}\right\rangle$ is the $\langle\mathrm{EE}\rangle$ expectation value for an ensemble of Monte Carlo simulations using the B03 instrument noise and the fiducial $\Lambda \mathrm{CDM}$ model as the CMB input. The quantities $\left\langle\mathcal{C}_{b}^{\mathrm{EE}}\right\rangle, \mathcal{C}_{b}$, and $\Delta \mathcal{C}_{b}$ are quoted in terms of $l(l+1) C_{l} /(2 \pi)$. $\langle\mathrm{EB}\rangle$ is not calculated for the $\Lambda \mathrm{CDM}$ shape, since it can go positive and negative. 
TABLE 4

Values of the $\Lambda$ Statistic and the PTE from the Comparison of the Wideband Band Powers when Compared to a Model with Zero Polarization Signal

\begin{tabular}{|c|c|c|c|c|c|c|c|}
\hline \multirow[b]{2}{*}{ SHAPE } & \multirow[b]{2}{*}{ MASK } & \multicolumn{2}{|r|}{$\langle\mathrm{EE}\rangle$} & \multicolumn{2}{|c|}{$\langle\mathrm{BB}\rangle$} & \multicolumn{2}{|c|}{$\langle\mathrm{EB}\rangle$} \\
\hline & & $\Lambda$ & PTE & $\Lambda$ & PTE & $\Lambda$ & PTE \\
\hline \multirow[t]{2}{*}{$l(l+1) C_{l} /(2 \pi) \ldots \ldots \ldots \ldots \ldots$} & NA 2 mask & 8.6 & $3.4 \times 10^{-5}$ & 0.42 & 0.36 & 2.2 & 0.04 \\
\hline & IT deep & 2.5 & 0.02 & 1.0 & 0.16 & $1.0 \times 10^{-3}$ & 0.97 \\
\hline$C_{l} \ldots \ldots \ldots$ & NA 2mask & 11.4 & $1.9 \times 10^{-6}$ & 0.30 & 0.44 & 0.94 & 0.17 \\
\hline$(2 l+1) C_{l} / 4 \pi \ldots \ldots \ldots \ldots \ldots \ldots$ & NA 2 mask & 10.7 & $3.7 \times 10^{-6}$ & 0.32 & 0.42 & 1.9 & 0.05 \\
\hline$\Lambda \mathrm{CDM}$ & NA 2 mask & 10.4 & $5.3 \times 10^{-6}$ & 0.61 & 0.27 & $\ldots$ & $\ldots$ \\
\hline
\end{tabular}

Notes.-Calculated from the data in Table 3. The $\langle\mathrm{EE}\rangle$ data is inconsistent with zero, while $\langle\mathrm{BB}\rangle$ and $\langle\mathrm{EB}\rangle$ are consistent with no polarization signal.

As discussed in Masi et al. (2006), each side of the focal plane has two PSB pairs at $145 \mathrm{GHz}$ that were oriented so that the left and right sides of the focal plane could measure Stokes $Q$ and $U$ independently. The (WX-YZ)/2 is done by taking the difference of maps made from the left (WX) and right (YZ) sides of the focal plane. In the same manner used for the $(h 1-h 2) / 2$ test, the NA pipeline makes separate (WX-YZ)/2 maps from the shallow and deep observations, while the IT pipeline makes a combined $(\mathrm{WX}-\mathrm{YZ}) / 2$ map from the deep and shallow data. For each pipe-
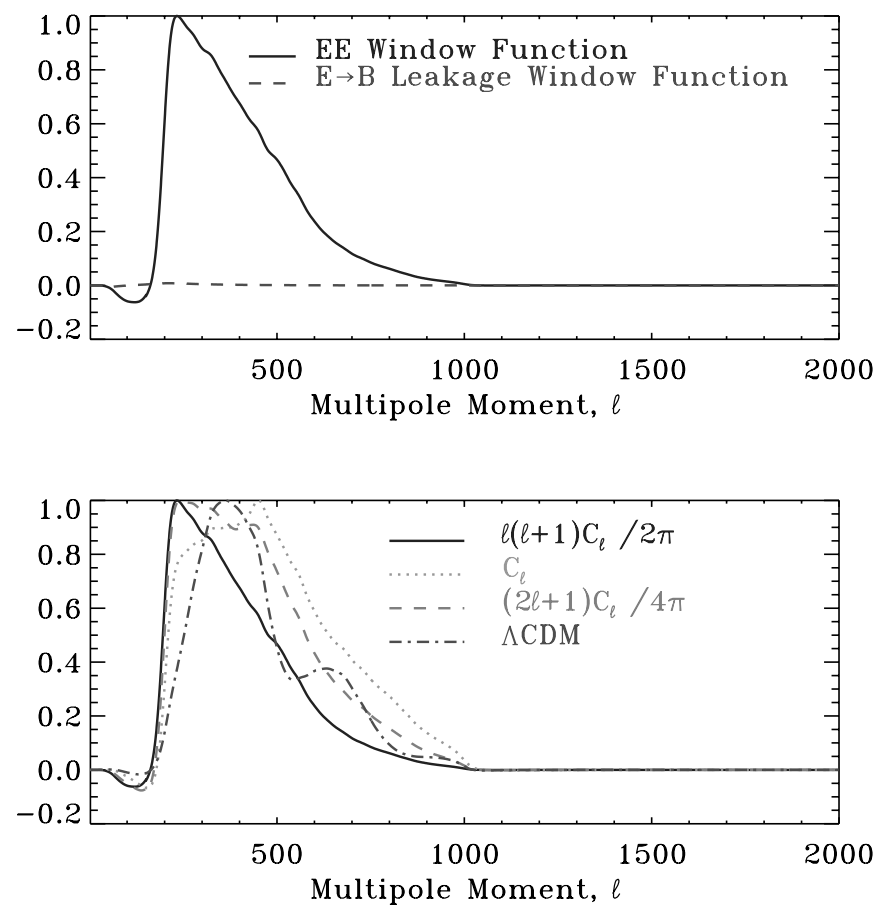

FIg. 3.-Window functions from the NA wideband results in Tables 3 and 4 . In the top panel the solid line is the $\langle\mathrm{EE}\rangle$ window function for the band $201 \geq l \leq 1000$. The dashed line characterizes the leakage of $E$-modes into $B$-modes $(E \rightarrow B)$, which has a maximum value of $\sim 0.01$. The $\langle\mathrm{BB}\rangle$ window function and $B \rightarrow E$ leakage window function are similar to those plotted here. The low amplitude of the $E \rightarrow B$ and $B \rightarrow E$ shows that $\mathrm{B} 03$ is able to separate $E$ - and $B$-mode polarization. In the bottom panel the window functions are shown for the different power spectrum parameterizations [i.e., $C_{l}^{(S)}$ ] used in the band power estimation. The shape of the $\langle E E\rangle$ window function indicates the effective weight applied to each multipole moment. For all cases used in the NA analysis, the window function is significantly different than the flat band used in the IT wideband analysis. This is due to an effective Wiener filter, which weights each multipole by $C_{l}^{(S)} /\left(C_{l}+N_{l}\right)^{2}$, where $N_{l}$ is the noise at a given multipole, $C_{l}^{(S)}$ is the shape function, and $C_{l}$ is the expected signal. In a given band the expected signal depends on the form of $C_{l}^{(S)}$. [See the electronic edition of the Journal for a color version of this figure.] line, the power spectra are estimated in the same way as in the $(h 1-h 2) / 2$ test.

Figure 4 shows the results for the $(h 1-h 2) / 2$ and the $(\mathrm{WX}-\mathrm{YZ}) / 2$ tests, and Table 5 shows $\chi^{2}$ and PTE's calculated from those results. For each pipeline, both jackknife tests are consistent with zero for all three spectra. These tests put strong limits on systematic problems.

\subsection{Simulation of Instrument Characterization Errors}

Mischaracterization of instrumental parameters is a potential source of systematic error in the power spectra. The primary parameters of concern are beam size, calibration, polarization efficiency, detector time constant, and polarization angle. An error in beam size leads to a bin-dependent scaling factor. Errors in the absolute calibration and polarization efficiency lead to an overall scaling factor. Errors in relative calibrations or detector time constants lead to leakage of CMB temperature anisotropies into

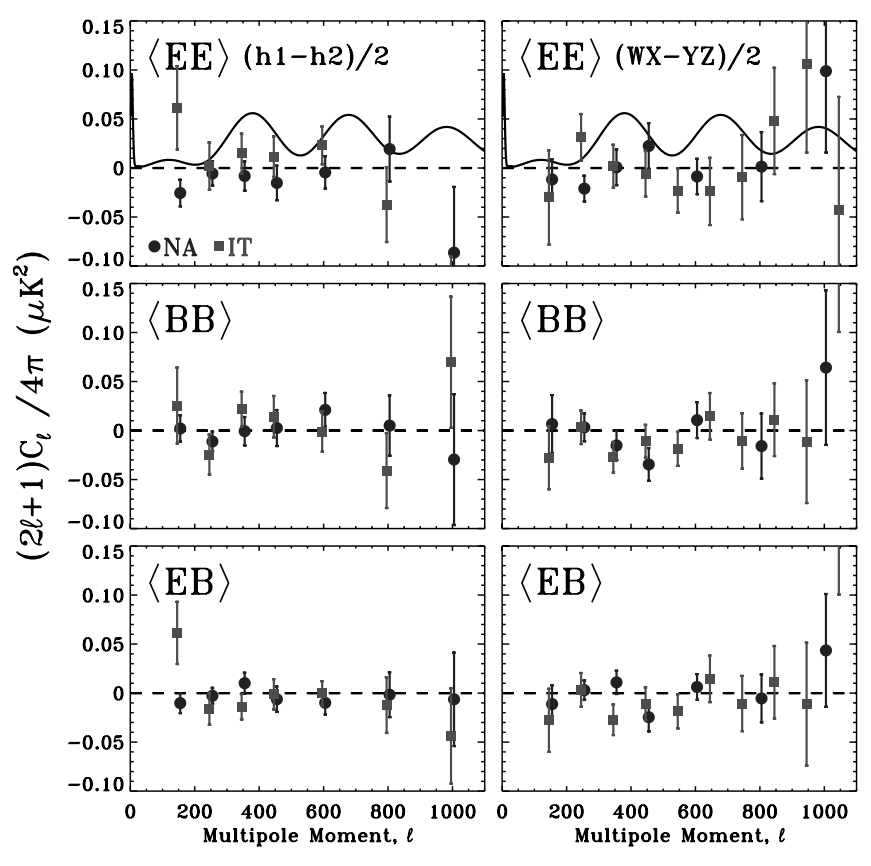

FIG. 4.- Results of jackknife tests. The left panels show the results for the $(h 1-h 2) / 2$ test, and the right panels show the results for the $(\mathrm{WX}-\mathrm{YZ}) / 2$ test. The circles are results from the NA pipeline, and the squares are results from the IT pipeline. Table 5 shows the $\chi^{2}$ and PTE calculated from these results. For both tests, all three spectra are consistent with zero signal. [See the electronic edition of the Journal for a color version of this figure.] 
TABLE 5

$\chi^{2}$ AND PTE fRom the $\mathbf{J}_{\text {ACKKnife Results }}$

\begin{tabular}{|c|c|c|c|c|}
\hline \multirow[b]{2}{*}{ SPECTRUM } & \multicolumn{2}{|c|}{ NA } & \multicolumn{2}{|c|}{ IT } \\
\hline & $\chi^{2}$ & PTE & $\chi^{2}$ & PTE \\
\hline \multicolumn{5}{|c|}{$(h 1-h 2) / 2$} \\
\hline$\langle\mathrm{EE}\rangle$ & 6.9 & 0.44 & 10.6 & 0.16 \\
\hline$\langle\mathrm{BB}\rangle$ & 3.0 & 0.89 & 6.0 & 0.54 \\
\hline$\langle\mathrm{EB}\rangle$ & 3.0 & 0.89 & 6.9 & 0.44 \\
\hline \multicolumn{5}{|c|}{$(\mathrm{WX}-\mathrm{YZ}) / 2$} \\
\hline$\langle\mathrm{EE}\rangle$ & 5.5 & 0.60 & 5.9 & 0.75 \\
\hline$\langle\mathrm{BB}\rangle$ & 6.7 & 0.46 & 1.5 & 0.997 \\
\hline$\langle\mathrm{EB}\rangle$ & 5.1 & 0.65 & 6.0 & 0.74 \\
\hline
\end{tabular}

Notes.-Calculated from results in Fig 4. Both pipelines have 7 degrees of freedom.

the polarization signal. An error in the polarization angle mixes the $Q$ and $U$ Stokes parameters.

The measurement of B03 instrument parameters is described in Masi et al. (2006), and the uncertainties on those parameters are shown here in Table 6 . To estimate the error induced by potential errors in relative calibration, time constant, polarization efficiency, and polarization angle, we performed a suite of signalonly Monte Carlo simulations. For each parameter we performed 145 simulations starting with the same simulated sky map (a realization of the fiducial $\Lambda C D M$ model). We then create a timeordered data stream in which the value of the parameter is randomly varied for each detector. The values are drawn from a distribution representing our uncertainty on that parameter. We then analyze this data stream using the measured parameter values. For each Monte Carlo we estimate the power spectrum using a technique similar to that used by the IT pipeline. We then compute the systematic error bar by taking the standard deviation of Monte Carlo results. Figure 5 shows the results of the simulations. The induced systematic error bars are less than $10 \%$ of the band power uncertainty. On most scales the polarization angle is the dominant source of error.

Although uncertainty in the beam size is a relatively benign problem, beam differences between elements in a PSB pair and structure in the cross-polar beam pattern of a given detector could lead to irreducible leakage of temperature anisotropies into the polarization maps. Given the off-axis structure of the BOOMERANG optics (where the axis of symmetry is vertical),

TABLE 6

Instrument Parameters, the Uncertainty on Their Characterization, and the Induced Error on the $\langle$ EE $\rangle$ Power Spectrum

\begin{tabular}{ccc}
\hline \hline Parameter & Uncertainty & Induced Error on Spectrum \\
\hline Beam FWHM................ & 0.23 & $2.5 \%$ at $l=500,10 \%$ at $l=1000$ \\
Absolute calibration ........ & $1.8 \%$ & $3.6 \%$ \\
Polarization efficiency..... & $3 \%$ & $4 \%$ \\
Relative calibration ......... & $0.4 \%$ & See Fig. 5 \\
Polarization angle ............ & $2 \%$ & See Fig. 5 \\
Time constant................ & $10 \%$ & See Fig. 5 \\
\hline
\end{tabular}

Notes.-Errors in beam size, absolute calibration, and polarization efficiency result in a rescaling of the power spectrum. Errors in relative calibration, polarization angle, and time constant are more complicated; see Fig. 5 for the induced error bars.

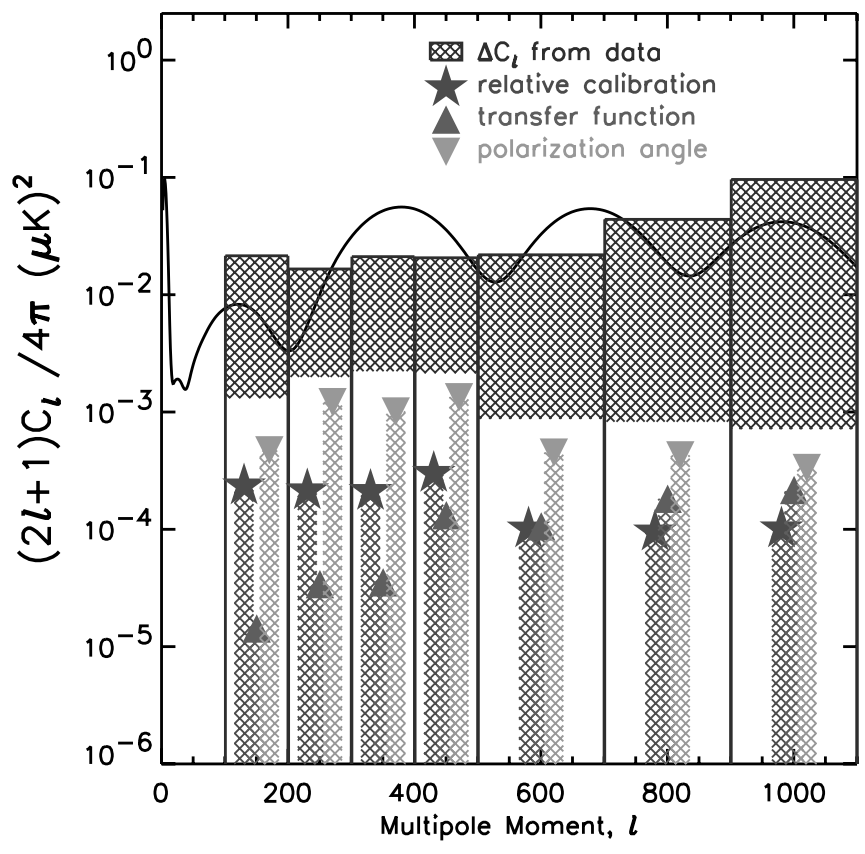

FIG. 5.-Propagation of measurement errors in instrumental parameters to $\langle E E\rangle$ error. The hatched bands show the upper edges of the $\langle E E\rangle$ error (due to noise) for the bands in the multibin NA results (Fig. 2). The other symbols show errors in relative calibration (stars), bolometer time constant (upward-pointing triangles) and polarization angle (downward-pointing triangles). Because errors in absolute calibration and polarization efficiency are multiplicative factors that act identically on each bin, their effect is left off this plot and reported instead in Table 6. [See the electronic edition of the Journal for a color version of this figure.]

the beam mismatch between elements in a PSB pair depends on the polarization angles of each element. For example, detectors oriented at $45^{\circ}$ and $-45^{\circ}$ with respect to the horizon have wellmatched beams, while detectors oriented at $0^{\circ}$ and $90^{\circ}$ will have slightly different beams. A physical optics simulation confirmed that the latter case has the worst mismatch among all four PSB pairs. For this worst case we calculate the differential beam window function and estimate the leakage of temperature anisotropy into polarization using the fiducial $\Lambda C D M$ model. We find this signal to be smaller than our measured $\langle\mathrm{EE}\rangle$ signal by a factor of $10^{3}$ (or more) on the angular scales that we are sensitive to. This calculation represents the worst-case scenario because sky rotation should reduce this contamination somewhat.

Differences between the cross-polar beams in a PSB pair could also lead to temperature anisotropy leakage. The integrated crosspolar beam for a given detector is a factor of $\sim 200$ smaller than the integrated co-polar beam. Here, if we take the worst-case scenario (i.e., that the throughput of cross-polar beam difference is twice the throughput of the cross-polar beam of one detector), a naive estimate of the $\langle\mathrm{TT}\rangle$ leakage into $\langle\mathrm{EE}\rangle$ gives $\langle\mathrm{EE}\rangle_{\text {leak }} \lesssim 10^{-4}\langle\mathrm{TT}\rangle$, which is $\lesssim 1 \%$ of the observed $\langle\mathrm{EE}\rangle$.

\section{FOREGROUNDS}

Polarized emission from Galactic and extragalactic sources are another potential source of contamination (de Oliveira-Costa 2004; Tucci et al. 2005). Currently, not much is known about diffuse polarized emission in the frequency range $30 \mathrm{GHz}<\nu<$ $400 \mathrm{GHz}$. Synchrotron emission is expected to be highly polarized, but the power steeply decreases with increasing frequency $(\alpha \sim-3)$. Recent observations with the Australian Telescope Compact Array have detected polarized synchrotron emission in a small patch near the edge of our deep region at frequencies of 
TABLE 7

Regions of Potential Dust Contamination

\begin{tabular}{llll}
\hline \hline \multicolumn{2}{c}{$\begin{array}{c}\text { R.A. Limits } \\
(\mathrm{deg})\end{array}$} & \multicolumn{2}{c}{$\begin{array}{c}\text { Decl. Limits } \\
(\mathrm{deg})\end{array}$} \\
\hline 84 & 85 & -48.5 & -47.25 \\
87.5 & 88.75 & -48.5 & -47.25 \\
87.5 & 88.5 & -49.5 & -50.5 \\
\hline
\end{tabular}

Notes.-Found by taking the difference between the B03 145 and $345 \mathrm{GHz}$ intensity maps. The polarization spectra were recalculated with the data in these regions excised. The resulting spectra are identical to those in Fig. 2 and Table 1.

$1.4 \mathrm{GHz}$ (Bernardi et al. 2003) and $2.3 \mathrm{GHz}$ (Carretti et al. 2006). A naive extrapolation of these synchrotron results to $145 \mathrm{GHz}$ predicts a signal of $0.2 \mu \mathrm{K}$ rms compared to a $\sim 3 \mu \mathrm{K}$ rms expected from the fiducial $\Lambda \mathrm{CDM}$ model given our beam.

From starlight polarization measurements (Fosalba et al. 2002), dust is expected to be less than $10 \%$ polarized, with a spectral index $\alpha \sim 1.7$, but it could be higher depending on the nature of the Galactic magnetic field (Wright 1987). Results from Archeops (Benoît et al. 2004; Ponthieu et al. 2005) measure a polarization fraction of $5 \%-10 \%$ for dust clouds near the Galactic plane, but the results are not sensitive enough to place strong limits on degree-scale dust polarization away from the Galactic plane.

From the $145 \mathrm{GHz}$ data alone, we are highly confident that our $E$-mode polarization signal is dominated by the $\mathrm{CMB}$ and not foreground emission. Foreground emission should produce nearly equal parts $E$ - and $B$-mode polarization. Nondetection of any $B$-mode signal (Fig. 2 and Tables 1-4) implies a lack of foreground polarization. In Piacentini et al. (2006) the B03 $\langle\mathrm{TB}\rangle$ signal is consistent with zero, while $\langle\mathrm{TE}\rangle$ is consistent with $\Lambda \mathrm{CDM}$. Further evidence is obtained by cross-correlating an IRAS dust intensity map with the $145 \mathrm{GHz}$ polarization data. Both the $\left\langle\mathrm{T}_{I R A S} \mathrm{~B}_{\mathrm{B} 03}\right\rangle$ and $\left\langle\mathrm{T}_{I R A S} \mathrm{E}_{\mathrm{B} 03}\right\rangle$ are consistent with zero. We find that the consistency of the deep-only and combined shallow+deep power spectra rule out large dust polarization signals in regions nearer to the Galactic plane.

In Masi et al. (2006), we characterize the dust emission by comparing the three B03 intensity maps to dust templates from Schlegel et al. (1998). In the deep region, we detect a dust intensity correlation at $2.5 \sigma$ with our $345 \mathrm{GHz}$ channels but find an upper limit of $4 \mu \mathrm{K}_{\mathrm{CMB}}$ rms for dust intensity at $145 \mathrm{GHz}$. If we assume that dust is $10 \%$ polarized, we get an upper limit of $0.4 \mu \mathrm{K}_{\mathrm{CMB}}$ rms for the dust polarization signal at $145 \mathrm{GHz}$. A polarized analysis of 245 and $345 \mathrm{GHz}$ data will be discussed in a future work.

Finally, the difference of the intensity maps at 145 and $345 \mathrm{GHz}$ shows what appears to be three small regions of diffuse dust emission, which appear to be correlated with IRAS emission (see Fig. 27 of Masi et al. 2006). As one final test, we perform a spectrum analysis on a sky cut in which we excised square blocks centered on these clouds. The boundaries of the blocks are reported in Table 7. The resulting polarized power spectra are identical to those reported here.

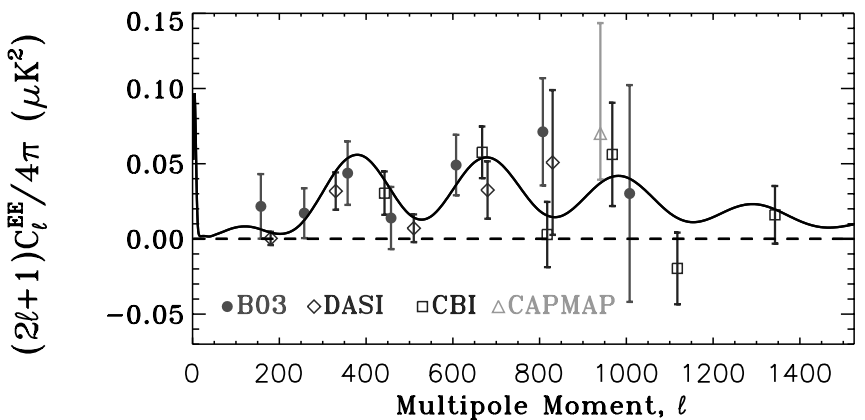

FIG. 6.-Comparison of B03 $\langle\mathrm{EE}\rangle$ results with existing results from DASI (Leitch et al. 2004), CBI (Readhead et al. 2004), and CAPMAP (Barkats et al. 2005). The model is the fiducial $\Lambda \mathrm{CDM}$ model. [See the electronic edition of the Journal for a color version of this figure.]

\section{CONCLUSIONS}

In this paper we report the measurement of the $\langle\mathrm{EE}\rangle,\langle\mathrm{BB}\rangle$, and $\langle\mathrm{EB}\rangle$ polarization power spectra from the 2003 flight of BOOMERANG. Reasonably consistent results have been obtained from two different data analysis pipelines. These results have passed a wide variety of systematic tests, and the induced error from instrumental uncertainties is negligible. The $\langle\mathrm{BB}\rangle$ and $\langle\mathrm{EB}\rangle$ results are consistent with zero signal, as expected in $\Lambda \mathrm{CDM}$ models dominated by scalar adiabatic perturbations. The $\langle\mathrm{EE}\rangle$ results are consistent with existing measurements (Fig. 6) and a good fit to the $\langle\mathrm{EE}\rangle$ signal expected from the $\Lambda \mathrm{CDM}$ model, which is the best fit to the $W M A P\langle\mathrm{TT}\rangle$ results. Several tests using higher frequency channels and dust maps, in addition to the fact that $\langle\mathrm{BB}\rangle$ and $\langle\mathrm{EB}\rangle$ are consistent with zero, argue that it is very unlikely that the $\langle\mathrm{EE}\rangle$ result is contaminated by Galactic emission. This detection of $\langle\mathrm{EE}\rangle$ is the first by a bolometric polarimeter and thus bodes well for the future of $\mathrm{CMB}$ polarimetry using bolometric detectors.

We gratefully acknowledge support from CIAR, CSA, and NSERC in Canada, ASI, University La Sapienza, and PNRA in Italy, PPARC and the Leverhulme Trust in the UK, and NASA (awards NAG5-9251 and NAG5-12723) and NSF (awards OPP 99-80654 and OPP 04-07592) in the US. Additional support for detector development was provided by CIT and the Jet Propulsion Laboratory (JPL). C. B. N. acknowledges support from a Sloan Foundation Fellowship; W. C. J. and T. E. M. were partially supported by NASA GSRP Fellowships. Field, logistical, and flight support was outstandingly supplied by USAP and NSBF; data recovery was especially appreciated. This research used resources at NERSC, supported by the DOE under contract DE-AC03-76SF00098, and the MacKenzie cluster at CITA, funded by the Canada Foundation for Innovation. We also thank the CASPUR Rome, Italy) computational facilities and the Applied Cluster Computing Technologies Group at JPL for computing time and technical support. Some of the results in this paper have been derived using the HEALPix (Górski et al. 2005) package, and nearly all have benefitted from the FFTW implementation of the discrete Fourier transform (Frigo \& Johnson 2005).
Barkats, D., et al. 2005, ApJ, 619, L127

Benoit, A., et al. 2004, A\&A, 424, 571

Bernardi, G., Carretti, E., Cortiglioni, S., Sault, R. J., Kesteven, M. J., \& Poppi, S. 2003, ApJ, 594, L5

Bond, J. R., Contaldi, C. R., \& Pogosyan, D. 2003, Philos. Trans. R. Soc. London A, 361, 2435

\section{REFERENCES}

Bond, J. R., \& Efstathiou, G. 1984, ApJ, 285, L45

Bond, J. R., Jaffe, A. H., \& Knox, L. 1998, Phys. Rev. D, 57, 2117 2000, ApJ, 533, 19

Bucher, M., Moodley, K., \& Turok, N. 2001, Phys. Rev. Lett., 87, 191301

Carretti, E., et al. 2006, MNRAS, 367, 132

Challinor, A., \& Chon, G. 2005, MNRAS, 360, 509 
Chon, G., Challinor, A., Hivon, E., Prunet, S., \& Szapudi, I. 2004, MNRAS, 350,914

Crill, B. P., et al. 2003, ApJS, 148, 527

Crittenden, R., Davis, R. L., \& Steinhardt, P. J. 1993, ApJ, 417, L13

de Oliveira-Costa, A. 2005, in ASP Conf. Ser. 343, Astronomical Polarimetry: Current Status and Future Directions, ed. A. Adamson, C. Aspin, \& C. J. Davis (San Fancisco: ASP), 485

Enqvist, K., \& Kurki-Suonio, H. 2000, Phys. Rev. D, 61, 043002

Fosalba, P., Lazarian, A., Prunet, S., \& Tauber, J. A. 2002, in AIP Conf. Proc. 609, Astrophysical Polarized Backgrounds, ed. S. Cecchini et al. (San Fransisco: ASP), 44

Frigo, M., \& Johnson, S. G. 2005, IEEE Proc., 93, 216

Górski, K. M., Hivon, E., Banday, A. J., Wandelt, B. D., Hansen, F. K., Reinecke, M., \& Bartelmann, M. 2005, ApJ, 622, 759

Hivon, E., Górski, K. M., Netterfield, C. B., Crill, B. P., Prunet, S., \& Hansen, F. 2002, ApJ, 567, 2

Hu, W. 2002, Phys. Rev. D, 65, 023003

Jones, W. C., Bhatia, R. S., Bock, J. J., \& Lange, A. E. 2003, Proc. SPIE, 4855, 227

Jones, W. C., et al. 2006a, ApJ, 647, 823 2006b, A\&A, submitted (astro-ph/0606606)

Kamionkowski, M., Kosowsky, A., \& Stebbins, A. 1997, Phys. Rev. D, 55, 7368

Kaplinghat, M., Knox, L., \& Song, Y.-S. 2003, Phys. Rev. Lett., 91, 241301

Knox, L. 1999, Phys. Rev. D, 60, 103516
Kogut, A., et al. 2003, ApJS, 148, 161

Kovac, J. M., Leitch, E. M., Pryke, C., Carlstrom, J. E., Halverson, N. W., \& Holzapfel, W. L. 2002, Nature, 420, 772

Leitch, E., et al. 2005, ApJ, 624, 10

MacTavish, C., et al. 2006, ApJ, 647, 799

Masi, S., et al. 2006, A\&A, submitted (astro-ph/0507509)

Netterfield, C. B., et al. 2002, ApJ, 571, 604

Piacentini, F., et al. 2002, ApJS, 138, 315 2006, ApJ, 647, 833

Pogosian, L., Vachaspati, T., \& Winitzki, S. 2002, Phys. Rev. D, 65, 083502 Polnarev, A. G. 1985, Soviet Astron, 29, 607

Ponthieu, N., et al. 2005, A\&A, 444, 327

Readhead, A. C. S., et al. 2004, Science, 306, 836

Rees, M. J. 1968, ApJ, 153, L1

Ruhl, J. E., et al. 2003, ApJ, 599, 786

Schlegel, D. J., Finkbeiner, D. P., \& Davis, M. 1998, ApJ, 500, 525

Seljak, U., \& Zaldarriaga, M. 1997, Phys. Rev. Lett., 78, 2054

Seljak, U., et al. 2005, Phys. Rev. D, 71, 103515

Spergel, D. N., et al. 2003, ApJS, 148, 175

Tucci, M., Martinez-Gonzalez, E., Vielva, P., \& Delabrouille, J. 2005, MNRAS, 360,935

Turner, M. S., \& White, M. 1996, Phys. Rev. D, 53, 6822

Wright, E. L. 1987, ApJ, 320, 818

Zaldarriaga, M., \& Seljak, U. 1997, Phys. Rev. D, 55, 1830 